\title{
Synthesis and characterization of mesoporous Si-MCM-41 materials and their application as solid acid catalysts in some esterification reactions
}

\author{
TARUN F PARANGI, RAJESH M PATEL and UMA V CHUDASAMA* \\ Applied Chemistry Department, Faculty of Technology and Engineering, M.S. University of Baroda, \\ Vadodara 390 001, India
}

MS received 9 October 2012; revised 25 December 2012

\begin{abstract}
Mesoporous MCM-41 has been synthesized by sol-gel method at room temperature possessing good thermal stability, high surface area as well as retention of surface area at high temperature. The MCM41 neutral framework has been modified and put to practical use by incorporating $\mathrm{Al}^{3+}$ in the siliceous $\mathrm{MCM}$ 41 framework and supporting 12-TPA (12-tungstophosphoric acid) onto MCM-41 by process of anchoring and calcination to induce Brønsted acidity in MCM-41 to yield Al-MCM-41 and 12TPA-MCM-41, respectively. The synthesized materials have been characterized for elemental analysis by ICP-AES, XRD, SEM, TEM, EDX, FT-IR and TGA. Surface area has been determined by BET method and pore size and pore size distribution determined by BJH method. Surface acidity has been evaluated by $\mathrm{NH}_{3}$-TPD method. The potential use of Al-MCM-41 and 12TPA-MCM-41 as solid acid catalysts has been explored and compared by studying esterification as a model reaction wherein monoesters such as ethyl acetate (EA), propyl acetate (PA), butyl acetate $(\mathrm{BA})$ and benzyl acetate $(\mathrm{BzA})$ have been synthesized, optimizing several parameters such as catalyst amount, reaction time, reaction temperature and mole ratio of reagents.
\end{abstract}

Keywords. Mesoporous material; Si-MCM-41; Al-MCM-41; heteropoly acid anchored onto MCM-41; esterification; solid acid catalyst.

\section{Introduction}

Esterification is an industrially important reaction for synthesis of plasticizers, perfumes, fragrance in cosmetics, flavours in food, diluents in paints and coatings and intermediates in drugs, dye stuffs and fine chemicals (Otera 2003). The conventional catalyst used in esterification reactions is sulphuric acid, methanesulfonic acid or $p$-toluenesulfonic acid that are cited as potential environmentally hazardous chemicals, that pose problems such as difficulty in handling, causing an acidic waste water, difficulty of catalyst recovery etc. (Ma et al 2003; Yadav and Murkute 2003). In view of the deficiencies encountered, there is a global effort to replace the conventional homogeneous liquid acids by heterogeneous solid acids. The use of solid acids, eliminates the corrosive action of liquid acids. Being heterogeneous in nature, separation from reaction mixture is easy and the catalyst can be regenerated and reused.

MCM-41 based materials have negligible catalytic activity due to framework neutrality, however, with advantageous properties like mesoporous nature of the

\footnotetext{
*Author for correspondence (uvcres@gmail.com)
}

material, good thermal stability, high surface area and retention of surface area at high temperatures. Thus, the main aim of the present study was to encash the advantageous properties of MCM-41 and enhance its practicability in the area of catalysis using Green Chemistry principles. There are a number of ways by which catalytic activity can be generated into the MCM-41 neutral framework. (i) Substitution of an $\mathrm{M}^{3+}$ cation, e.g. $\mathrm{Al}^{3+}$ in the $\mathrm{Si}^{4+}$ framework, leading to negatively charged framework, followed by balancing these charges by $\mathrm{H}^{+}$ions (via $\mathrm{NH}_{4}^{+}$ion exchange and subsequent thermal decomposition to give $\mathrm{H}^{+}$and $\mathrm{NH}_{3}$ ) to create Brønsted acid sites. (ii) Immobilization/anchoring/impregnation of homogenous acid catalyst, e.g. heteropoly acids (HPAs) onto MCM-41. Heteropoly acids (HPAs) have proved to be the alternative to traditional mineral acid catalysts due to both strong acidity and appropriate redox properties. However, limitations for HPAs to be used as solid acid catalysts are low thermal stability, low surface area (1$10 \mathrm{~m}^{2} / \mathrm{g}$ ) and difficulty in separation from reaction mixture due to their high solubility in polar solvents (Beck et al 1992). For HPAs to be effective as catalysts, they should be supported on a carrier with a large surface area. Owing to a very large surface area and a uniform large pore size, the MCM-41 materials can act as excellent 
supports that provide an opportunity for HPAs to be dispersed over a large surface area and hence increased catalytic activity.

Several workers have used MCM-41-based materials as solid acid catalysts for esterification. Catalytic esterification of oleic acid over $\mathrm{SO}_{4}^{2-} / \mathrm{MCM}-41$ (Nascimento et al 2011), use of mesoporous silica supported diarylammonium catalysts for esterification of free fatty acid in greases (Helen et al 2010), application of sulfated zirco$\mathrm{nia} /$ hexagonal mesoporous silica (HMS) catalyst in the esterification of gossypol (Zhu et al 2012), use of 12TPA (12-tungstophosphoric acid) supported onto $\mathrm{SnO}_{2}$ for the production of biodiesel (Lingaiah et al 2011) and esterification of acetic acid with ethyl alcohol over 12TPA supported on silica (Said et al 2007) have been reported.

The present study involves the synthesis of siliceous mesoporous MCM-41 at room temperature by sol-gel method, using templates. Further, $\mathrm{Al}^{3+}$ has been incorporated in the siliceous MCM-41 framework and 12-TPA (where 12-TPA = 12-tungstophosphoric acid, a HPA) has been supported onto MCM-41 by process of anchoring and calcination to induce Brønsted acidity in MCM-41 to yield Al-MCM-41 and 12TPA-MCM-41, respectively.

All synthesized materials have been characterized for elemental analysis by ICP-AES, X-ray diffraction (XRD), transmission electron microscopy (TEM), scanning electron microscopy (SEM), energy-dispersive X-ray spectroscopy (EDX), surface area (BET method), pore volume and pore distribution (BJH method), surface acidity by temperature programmed desorption (TPD) of ammonia, diffuse reflectance spectroscopy (UV-DRS), Fourier-transform infrared spectroscopy (FT-IR) and thermogravimetric analysis (TGA).

The potential use of Al-MCM-41 and 12TPA-MCM-41 as solid acid catalysts has been explored and compared by studying esterification as a model reaction wherein monoesters such as ethyl acetate (EA), propyl acetate (PA), butyl acetate (BA) and benzyl acetate (BzA) have been synthesized, optimizing several parameters such as catalyst amount, reaction time, reaction temperature and mole ratio of reagents.

\section{Experimental}

\subsection{Materials}

Commercial grade sodium silicate $\left(\mathrm{Na}_{2} \mathrm{SiO}_{3}\right)$ with composition, $28 \% \mathrm{SiO}_{2}$ and $7.5 \% \mathrm{Na}_{2} \mathrm{O}$ was procured from Sapna Chemicals, Vadodara. Cetyl trimethyl ammonium bromide $(\mathrm{CTABr})$, cetyl pyridinium bromide $(\mathrm{CPBr})$, sodium hydroxide flakes, aluminium sulfate, ammonium nitrate and 12-tungstophosphoric acid (12-TPA) were purchased from Loba Chemicals, Mumbai. Tetra ethylortho silicate (TEOS) and analytical grade sulphuric acid were obtained from E Merck, Mumbai. All other chemicals and reagents used were of analytical grade. Doubledistilled water (DDW) was used for all the studies.

\subsection{Catalyst synthesis}

2.2a Synthesis of MCM-41: MCM-41 has been synthesized by sol-gel method using templates varying several parameters such as silica source, templating agent/types, reaction conditions such as $\mathrm{pH}$, time of reaction, aging, temperature, etc., and these parameters are optimized, using surface area as an indicative tool in all cases. Table 1 - ESI describes the parameters that have been optimized for synthesis of MCM-41.

2.2b Synthesis of MCM-41 at optimized condition: The molar gel composition for MCM-41 is $1 \mathrm{SiO}_{2}: 0.5$ CTABr: $0 \cdot 25 \mathrm{Na}_{2} \mathrm{O}: 80 \mathrm{H}_{2} \mathrm{O}$. The first step was preparation of the precursor solution. $63.02 \mathrm{~g}$ of $\mathrm{Na}_{2} \mathrm{SiO}_{3}$ was mixed with $183 \mathrm{~g}$ DDW under continuous stirring at room temperature for $\sim 15 \mathrm{~min}$ in a polypropylene container (A). An aqueous solution of CTABr was prepared by dissolving 54.14 g CTABr in $200 \mathrm{~g}$ DDW under continuous stirring at room temperature (B). Template solution (B) was added to precursor solution (A) dropwise and with continuous stirring for $\sim 15 \mathrm{~min}$ and the solution further stirred for $15 \mathrm{~min}$. The $\mathrm{pH}$ of the resultant solution was adjusted to $\sim 10 \cdot 5$ using $1: 1 \mathrm{H}_{2} \mathrm{SO}_{4}$ (diluted $1: 1 \mathrm{v} / \mathrm{v})$. A gel was obtained at this stage which was stirred further for $30 \mathrm{~min}$. The polypropylene container was now closed and allowed to age at room temperature without stirring for $24 \mathrm{~h}$. The resultant gel was filtered, washed with DDW to remove adhering ions and dried at $120{ }^{\circ} \mathrm{C}$, followed by calcination at $550{ }^{\circ} \mathrm{C}$ for $6 \mathrm{~h}$ at a heating rate of $2{ }^{\circ} \mathrm{C} / \mathrm{min}$. The final material obtained was used for all further studies.

2.2c Synthesis of Al-MCM-41: In the present synthetic endeavour the objective is to synthesize mesoporous Al-MCM-41 at room temperature with good thermal stability and high surface acidity. A sol-gel method has been used to achieve this objective. Several sets of materials were prepared varying silica to alumina ratios, apart from other conditions, where surface acidity has been used as an indicative tool in all cases. Table 2 - ESI describes parameters that have been optimized for synthesis of Al-MCM-41.

2.2d Synthesis of Al-MCM-41 at optimized condition: The molar gel composition of Al-MCM-41 is $1 \mathrm{SiO}_{2}$ : 0.033 $\mathrm{Al}_{2} \mathrm{O}_{3}: 0.4 \mathrm{CTABr}: 0.25 \mathrm{Na}_{2} \mathrm{O}: 90 \mathrm{H}_{2} \mathrm{O}$. The first step was preparation of the precursor solution. $57.48 \mathrm{~g}$ of $\mathrm{Na}_{2} \mathrm{SiO}_{3}$ was mixed with $197.5 \mathrm{~g}$ DDW under continuous stirring at room temperature for $\sim 15 \mathrm{~min}$, in a polypropylene container, to which an aqueous solution of 
Table 1. \% yields of mono esters using 12TPA-MCM-41-20 and Al-MCM-41-30 at optimized condition.

\begin{tabular}{|c|c|c|c|c|c|c|}
\hline \multirow[b]{2}{*}{ S. no. } & \multirow[b]{2}{*}{ Reactants } & \multirow[b]{2}{*}{ Product } & \multicolumn{2}{|c|}{ 12ТРА-МСМ-41-20 } & \multicolumn{2}{|c|}{ Al-MCM-41-30 } \\
\hline & & & $\%$ Yield & *TON & $\%$ Yield & $* \mathrm{TON}$ \\
\hline 1 & $\mathrm{Bz}+\mathrm{AA}$ & $\mathrm{BzA}$ & $95 \cdot 23$ & $62 \cdot 84$ & $93 \cdot 55$ & 61.73 \\
\hline 2 & $\mathrm{E}+\mathrm{AA}$ & EA & $92 \cdot 31$ & $41 \cdot 83$ & $90 \cdot 35$ & $40 \cdot 94$ \\
\hline 3 & $\mathrm{P}+\mathrm{AA}$ & PA & $90 \cdot 23$ & $45 \cdot 09$ & $88 \cdot 25$ & $44 \cdot 10$ \\
\hline 4 & $\mathrm{~B}+\mathrm{AA}$ & BA & $74 \cdot 13$ & $40 \cdot 51$ & $73 \cdot 16$ & 39.98 \\
\hline A & Reusability of catalyst & & & & & \\
\hline 5 & $\mathrm{Bz}+\mathrm{AA} 1$ st cycle & BzA & $87 \cdot 15$ & $57 \cdot 51$ & $89 \cdot 73$ & $59 \cdot 21$ \\
\hline 6 & $\mathrm{Bz}+\mathrm{AA} 2$ nd cycle & BzA & $77 \cdot 45$ & $51 \cdot 10$ & $83 \cdot 80$ & $55 \cdot 29$ \\
\hline 7 & $\mathrm{E}+\mathrm{AA} 1$ st cycle & EA & $87 \cdot 10$ & $39 \cdot 46$ & $85 \cdot 20$ & $38 \cdot 60$ \\
\hline 8 & $\mathrm{E}+\mathrm{AA}$ 2nd cycle & EA & $78 \cdot 65$ & $35 \cdot 64$ & $79 \cdot 15$ & $35 \cdot 86$ \\
\hline 9 & $\mathrm{P}+\mathrm{AA} 1$ st cycle & PA & $84 \cdot 42$ & $42 \cdot 19$ & $85 \cdot 41$ & $42 \cdot 86$ \\
\hline 10 & $\mathrm{P}+\mathrm{AA}$ 2nd cycle & PA & $78 \cdot 35$ & $39 \cdot 15$ & $80 \cdot 25$ & $40 \cdot 10$ \\
\hline 11 & B + AA 1st cycle & BA & $69 \cdot 00$ & $37 \cdot 71$ & $69 \cdot 45$ & $37 \cdot 95$ \\
\hline 12 & $\mathrm{~B}+\mathrm{AA}$ 2nd cycle & BA & $61 \cdot 48$ & $33 \cdot 60$ & $64 \cdot 32$ & $35 \cdot 15$ \\
\hline
\end{tabular}

$\mathrm{Bz}=$ benzyl alcohol; $\mathrm{AA}=$ acetic acid $\mathrm{E}=$ ethanol; $\mathrm{P}=1$-propanol $; \mathrm{B}=1$-butanol; mole ratio of reactants = $1.5: 1$ (acid : alcohol); reaction time $=8 \mathrm{~h}$. Catalyst amount $=0.15 \mathrm{~g}$; reaction temperature $80{ }^{\circ} \mathrm{C}$ for EA and $115^{\circ} \mathrm{C}$ for PA, BA and BzA; *TON = turn over number, gram of ester formed per gram of catalyst.

aluminium sulfate was added (prepared by dissolving $5.643 \mathrm{~g}$ aluminium sulfate in $40 \mathrm{~g}$ DDW) dropwise and with constant stirring for $\sim 15 \mathrm{~min}$. This is the precursor solution (A). An aqueous solution of CTABr was prepared by dissolving $39.5 \mathrm{~g} \mathrm{CTABr}$ in $160 \mathrm{~g}$ DDW under continuous stirring at room temperature (B). Template solution (B) was added to precursor solution (A), dropwise and under constant stirring for $\sim 15 \mathrm{~min}$. The $\mathrm{pH}$ of the resultant solution was adjusted to $\sim 10.5$ using $1: 1$ $\mathrm{H}_{2} \mathrm{SO}_{4}$ (diluted 1:1 v/v). A gel was formed which was further stirred for $30 \mathrm{~min}$. The polypropylene container was now closed and allowed to age at room temperature without stirring for $24 \mathrm{~h}$. The resultant gel was filtered, washed with DDW to remove adhering ions and dried at $120^{\circ} \mathrm{C}$ followed by calcination at $550{ }^{\circ} \mathrm{C}$ for $6 \mathrm{~h}$, at a heating rate of $2{ }^{\circ} \mathrm{C} / \mathrm{min}$. After thermal treatment, the samples with various silica: alumina ratios Al-MCM-41-20, Al-MCM-41-30, Al-MCM-41-50 and Al-MCM-41-100 were subjected to ion exchange by treating them with aqueous $1.0 \% \mathrm{NH}_{4} \mathrm{NO}_{3}$ solution under continuous stirring for $3 \mathrm{~h}$, followed by calcination at $550{ }^{\circ} \mathrm{C}$ for $3 \mathrm{~h}$ at a heating rate of $2{ }^{\circ} \mathrm{C} / \mathrm{min}$ in air flow. The sample, Al-MCM-41-30 exhibits maximum surface acidity and was used for all further studies (table 3 - ESI).

For synthesis described in $\$ \$ 2.2 .3$ and 2.2.4 above, $\mathrm{Na}_{2} \mathrm{SiO}_{3}$ as a silica source is preferred to TEOS, reasons being higher cost of TEOS, thermal stability of final material obtained is better and excess negative charge developed in the framework due to substitution of $\mathrm{Si}^{4+}$ by $\mathrm{Al}^{3+}$, requires $\mathrm{Na}^{+}$for charge balance. While preparation, it is preferable to add template to precursor source. The $\mathrm{pH}$ of template being almost neutral and $\mathrm{pH}$ of precursor being $>12$, if template is added to precursor source, the $\mathrm{pH}$ variation window is narrowed down due to which $\mathrm{pH}$ of gel formation is easily adjusted. The $\mathrm{pH}$ in the synthe- sis was adjusted to $\sim 10.5$ because gel viscosity is maximum at this $\mathrm{pH}$, which can also be stirred with ease for homogenization. Further, in Al-MCM-41 synthesis, lower the $\mathrm{SiO}_{2}: \mathrm{Al}_{2} \mathrm{O}_{3}$ ratio, the higher will be $\mathrm{Al}^{3+}$ content and hence better is the acidity generated in the resulting materials. At the optimized condition surface acidity is high as well as surface area retention between $550-900{ }^{\circ} \mathrm{C}$ is fairly good.

2.2e Synthesis of 12-TPA supported MCM-41: The aim was to load different wt $\%$ of 12-TPA onto MCM-41 and induce acidity into the material, using surface acidity as an indicative tool in all cases. Four samples (12-TPA supported onto MCM-41) of the catalyst were prepared with varying 12 -TPA loading (10-40 wt\%). In a typical setup (10\% 12-TPA loading), $1 \mathrm{~g}$ of 12-TPA was dissolved in $100 \mathrm{ml} \mathrm{DDW}$, to which $9 \mathrm{~g}$ of MCM-41 was added (synthesized as described earlier in the text), and the resultant slurry stirred continuously for $24 \mathrm{~h}$ at room temperature. The excess solution was removed under vacuum, dried and subsequently calcined at $350{ }^{\circ} \mathrm{C}$ for $2 \mathrm{~h}$ at a heating rate of $2{ }^{\circ} \mathrm{C} / \mathrm{min}$.

\subsection{Instrumental methods of analysis}

All syntheized materials were subjected to instrumental methods of analysis/characterization. Elemental analysis was performed on ICP-AES spectrometer (Thermo-Scientific iCAP 6000 series). X-ray diffractogram $(2 \theta=$ $1-40^{\circ}$ ) was obtained on X-ray diffractometer (Bruker D8 Focus) with $\mathrm{CuK} \alpha$ radiation with nickel filter. FT-IR spectra was recorded using $\mathrm{KBr}$ pellet on Shimadzu (Model 8400S). Thermal analysis (TGA) was performed on a Shimadzu (Model TGA 50) thermal analyzer at a heating rate of $10^{\circ} \mathrm{C} \mathrm{min}^{-1}$. SEM and EDX of the sample 
were scanned on Jeol JSM-5610-SLV scanning electron microscope. TEM was performed using Philips CM30 ST electron microscope operated at $300 \mathrm{kV}$. Surface area measurements were determined using Micromeritics Gemini at $-196^{\circ} \mathrm{C}$ using nitrogen adsorption isotherms. Surface acidity was determined by $\mathrm{NH}_{3}$-TPD method using Micromeritics Chemisorb 2720. UV-Vis diffuse reflectance spectra was obtained using Shimadzu (Model UV-DRS 2450).

\subsection{Synthesis of monoesters (EA, PA, BA and BzA)}

In a typical reaction, a $100 \mathrm{~mL}$ round bottomed flask equipped with a Dean and Stark apparatus, attached to a reflux condenser was used and charged with acetic acid $(0 \cdot 05-0 \cdot 10 \mathrm{M})$, alcohol $(0 \cdot 05-0 \cdot 10 \mathrm{M})$, catalyst $(0 \cdot 10-$ $0 \cdot 20 \mathrm{~g})$ and a suitable solvent $(15 \mathrm{~mL})$. Cyclohexane was used as a solvent for the synthesis of ethyl acetate and toluene for propyl acetate, butyl acetate and benzyl acetate. The reactions were carried out varying several parameters such as amount of catalyst, mole ratio of reactants, reaction time, and these parameters optimized. After completion of reaction, catalyst was separated by decantation and reaction mixture was distilled to obtain the product.

\subsection{Regeneration of catalyst}

During the course of the reaction, there is a possibility of adsorption of reacting molecules on to the surface of the catalyst. After separation of catalyst in reaction mixture by decantation, it is first refluxed in ethanol for $30 \mathrm{~min}$ to solubilize and remove adsorbed molecules, followed by drying at $120^{\circ} \mathrm{C}$. This material was used as recycled catalyst. This regeneration procedure was followed in subsequent recycle reaction.

\section{Result and discussion}

\subsection{Catalyst characterization}

The characterization of MCM-41 and Al-MCM-41 are well investigated and presented in ESI (figures 1-19) which include XRD, TEM, SEM, EDX, FT-IR, TGA, pore size and pore volume distribution, surface are using nitrogen adsorption isotherm, surface acidity ( $\left.\mathrm{NH}_{3}-\mathrm{TPD}\right)$ and UV-DRS. The characterization indicate mesoporous nature of the materials.

12-TPA supported onto MCM-41 with 12-TPA loading $=20 \mathrm{wt} \%$, abbreviated as 12TPA-MCM-41-20 has been used for characterization, as it exhibits highest surface acidity and used for all catalytic studies.

The three main reliable techniques used for characterization of 12TPA-MCM-41-20 are XRD (Pandurangan et al 2005; Yarmo et al 2007), FT-IR and surface acidity which ensure the anchoring of HPA's on to MCM-41, wherein comparisons are made between pure HPA's, pure MCM-41 and a combination of these.

XRD of different wt\% loading of 12-TPA-MCM-41 are presented in figure 1. With reference to MCM-41 (a) and 12-TPA (f); (b), (c), (d) and (e) shows that 12-TPA is loaded on to MCM-41. (e) with the highest loading shows that the 12-TPA remains on the surface and the X-ray pattern is close towards (f). The observed results coincide well with those reported in literature (Pandurangan et al 2005; Yarmo et al 2007).

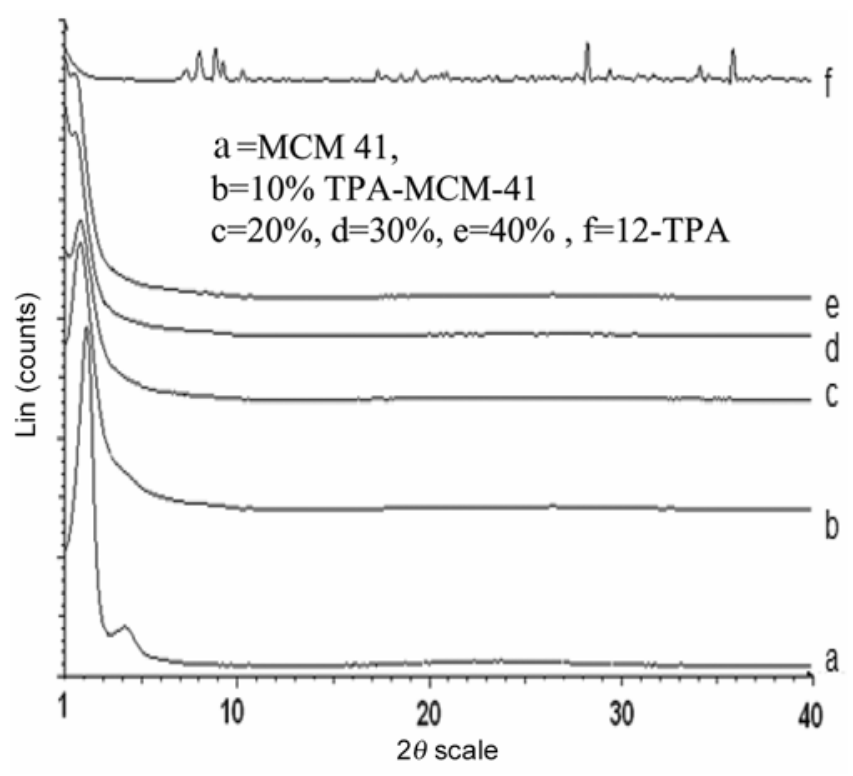

Figure 1. XRD of 12TPA-MCM-41.

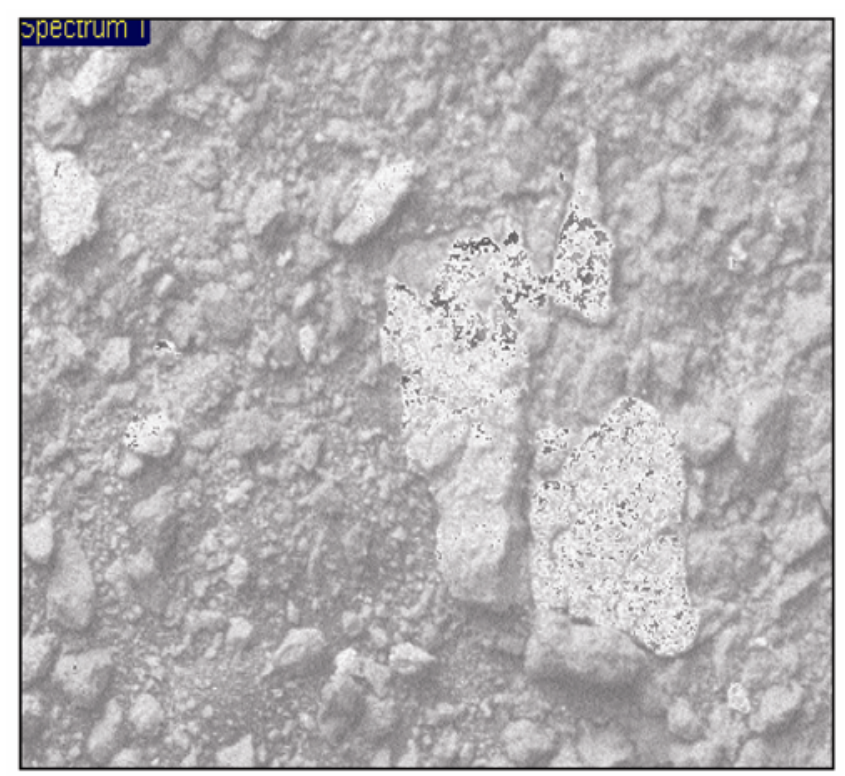

Figure 2. SEM of 12TPA-MCM-41-20. 
SEM image of 12TPA-MCM-41-20 (figure 2) exhibits irregular particle size. EDX of 12TPA-MCM-41-20, presented in figure $20-$ ESI shows the presence of W atomic $\%=2 \cdot 20, \mathrm{Si}$ atomic $\%=30 \cdot 44$ and $\mathrm{O}$ atomic $\%=$ 67.40. Absence of $\mathrm{P}$ in EDX is probably due to very low $\%$ of $\mathrm{P}$ in the original compound, which is probably not detected in EDX due to instrument limitations.

FT-IR spectra of HPA supported on to MCM-41 have been discussed earlier (Bekkum et al 1995; Edler and White 1997; Ma et al 1997; Xia et al 2000). FT-IR spectra (figure 3 ) with different 12-TPA loading coincide well with those reported in literature (Bekkum et al 1995; Edler and White 1997; Ma et al 1997; Xia et al 2000).

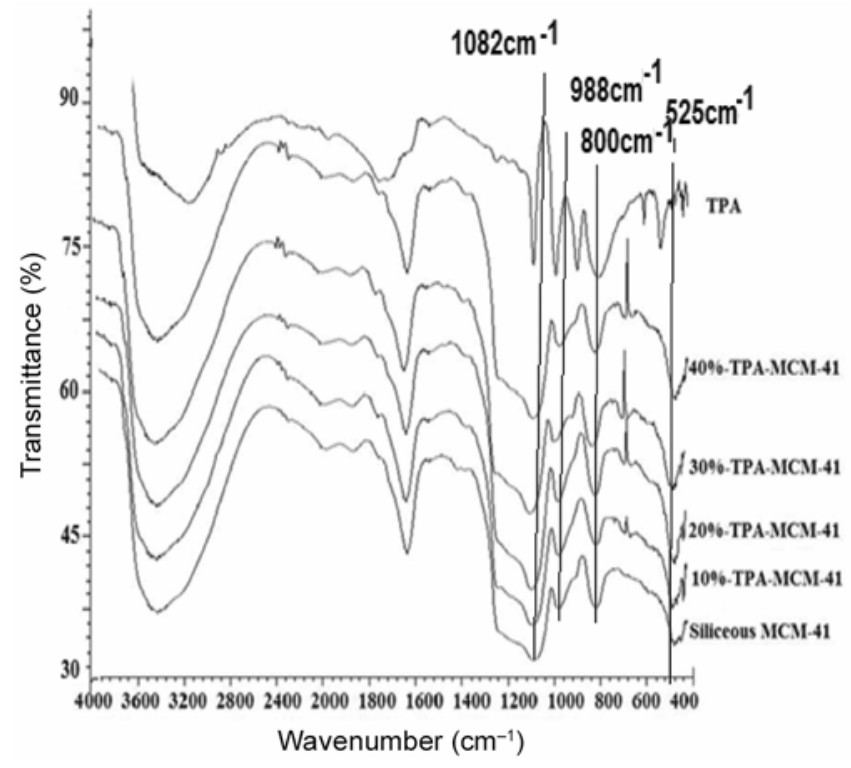

Figure 3. FT-IR of 12TPA-MCM-41.

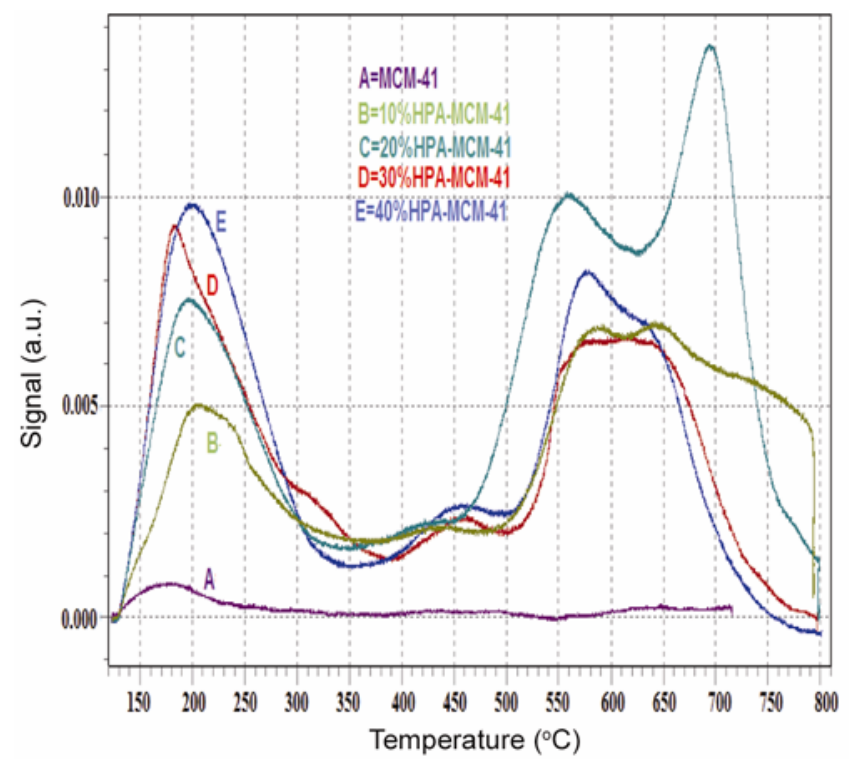

Figure 4. Ammonia TPD of 12TPA-MCM-41.
Comparative TPD pattern of MCM-41 samples with different 12-TPA loading (figure 4) exhibits two distinct peaks at 200 and $600{ }^{\circ} \mathrm{C}$ indicating presence of medium and strong acid sites, respectively, in all the samples. The surface acidity increases gradually with increasing 12TPA loading from 10 to $20 \mathrm{wt} \%$, after which a decrease is observed (table 3 - ESI).

\subsection{Synthesis of monoesters}

Equilibrium constants of the esterification reactions are low. As in any equilibrium reaction, the reaction may be driven to the product side by controlling the concentration of one of the reactants (Le Chatlier's principle). When concentration of one of the reactant relative to the other is increased, the reaction is driven to the product side. In order to obtain higher yield of esters, Le Chatlier's principle has been followed. Solvents cyclohexane and toluene have been employed to remove the water

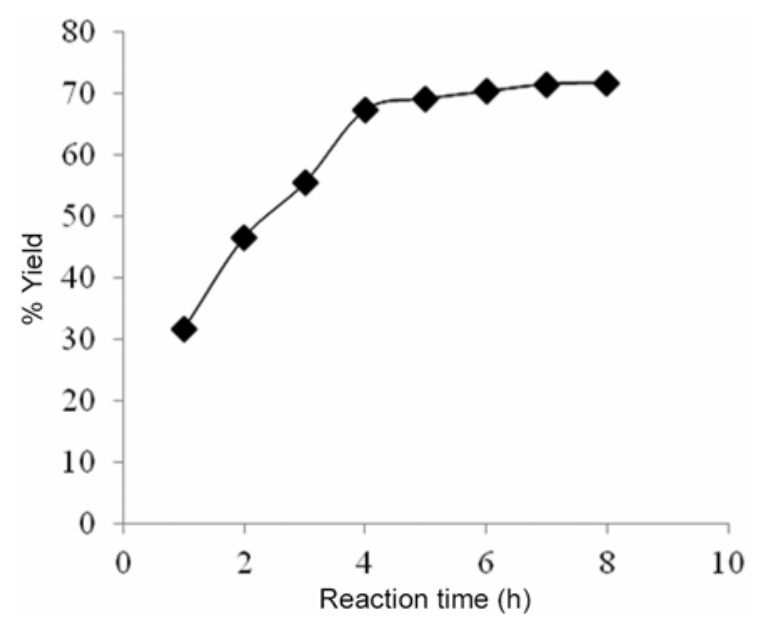

Figure 5. Reaction time variations for BzA synthesis.

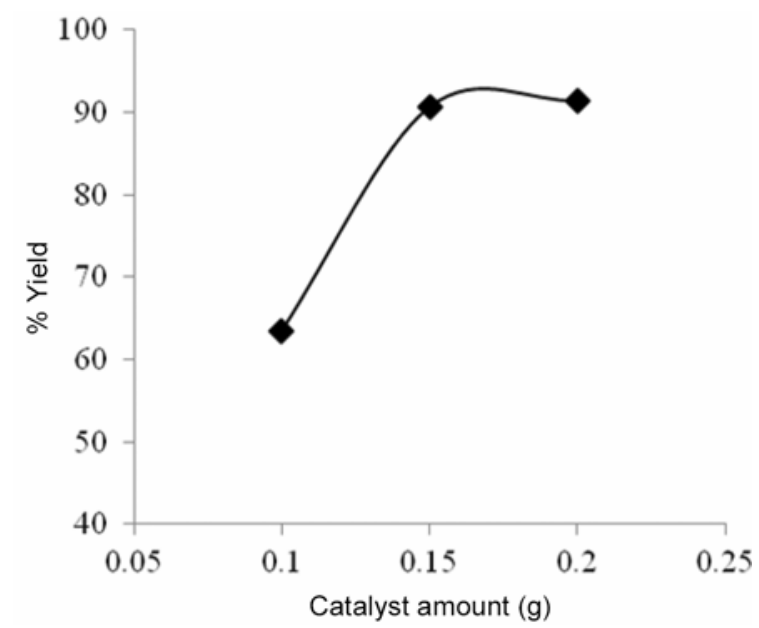

Figure 6. Catalyst amount variations for BzA synthesis. 
formed during the reaction as a binary azeotrope. Mono esters EA, PA, BA and BzA were synthesized as described in experimental section.

Firstly, reaction conditions were optimized using 12TPA-MCM-41-20 as solid acid catalyst for BzA synthesis by varying parameters such as catalyst amount, initial mole ratio of the reactant (alcohol to acid) and reaction time, and the results obtained presented in table 4 - ESI and a graphical presentation (figures 5 and 6).

During optimization of reaction time, it is observed that the conversion rate was very high initially, indicating that the reaction obeys first order kinetics. As reaction time increases, percentage yield increases. However, there is not much gain in product after $8 \mathrm{~h}$. Hence, the optimum reaction time was selected as $8 \mathrm{~h}$. With increasing amount of the catalyst, the $\%$ yield increased which is due to proportional increase in the number of active sites. The influence of reactant ratio (alcohol: acid) was studied by increasing from $1: 1 \cdot 5$ to $1 \cdot 5: 1$. The yield can be increased by increasing the concentration of either alcohol or acid. As observed from table $4-$ ESI, the \% yield of ester increases with increase in mole ratio of acid, while decreases with increasing mole ratio of alcohol. This may be attributed to preferential adsorption of alcohol on the catalyst which results in blocking of active sites. For economic reasons also, the reactant that is usually less expensive of the two, is taken in excess. In the present study, acids were used in excess. The temperature parameter has not been varied, due to the fact that the reaction temperature is sensitive to boiling points of reactants as well as solvents used as azeotrope.

At the optimum condition (mole ratio of reactants, alcohol $:$ acid $=1: 1 \cdot 5$, amount of catalyst $=0 \cdot 15 \mathrm{~g}$, time $=$ $8 \mathrm{~h}$ ), mono esters EA, PA and BA have been synthesized and the yields of esters are presented in table 1. Further,

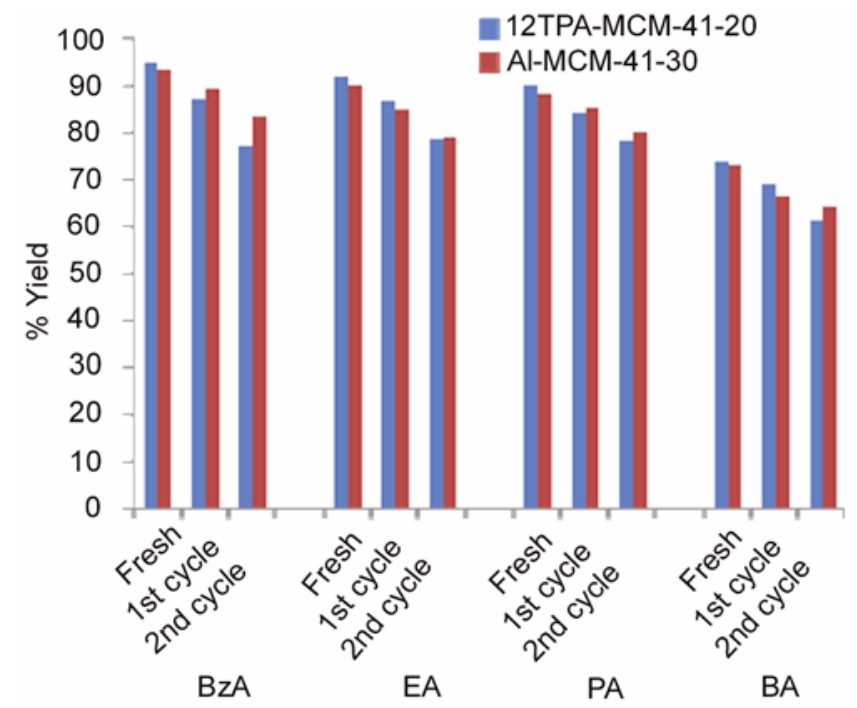

Figure 7. Comparative catalyst performance in formation of mono esters. for comparative study, Al-MCM-41-30 is used as solid acid catalyst for synthesis of mono esters BzA, EA, PA and $\mathrm{BA}$ at the above mentioned optimum condition. The results are presented in table 1 .

It is observed that the order of $\%$ yield of ester formed for both catalysts is $\mathrm{BzA}>\mathrm{EA}>\mathrm{PA}>\mathrm{BA}$. Though the yields of mono esters using both catalysts are comparable, higher yields are observed in case of 12TPA-MCM41-20 which could be attributed to higher surface acidity. Turn over number (TON) reflects the effectiveness of a catalyst and this also follows the order of ester formation.

Esterification of mono esters EA, PA and BA has been reported (Zhao 2001) in absence of catalyst and exhibit poor yields. Therefore, catalyst is a must for these reactions. In case of BzA, however, it is observed that with an excess of acetic acid and in the absence of any catalyst the yield is as high as $90.6 \%$ which is attributed to auto catalysis. In another report (Sharath et al 2001), high yields of BzA were obtained with small amount of the catalyst, but the reaction time was relatively high. In the present study, yields of BzA are $\sim 95 \%$ for both catalysts compared to above literature reports. Higher yields in case of benzyl acetate could be attributed to an enhanced nucleophilicity due to presence of aromatic ring in benzyl alcohol. The order of $\%$ yield of ester formed is EA > PA > BA could be explained due to increase in carbon chain length in the respective alcohols used for ester formation. During condensation of these alcohols with acetic acid, probably steric effects are responsible for explaining decreasing yields from EA through PA to BA.

$\%$ of yields obtained in recycled catalyst and \% decrease in yields in subsequent cycles is presented in tables 1 and 5 (ESI), respectively, and a graphical presentation (figure 7). It is observed that in subsequent cycles, decrease in \% yields is less for Al-MCM-41-30 compared to 12TPA-MCM-41-20. This could probably be due to the leaching of 12-TPA from surface of MCM-41. It is observed that the colour of the catalyst changes after each catalytic run. This gives an indication that during the course of the reaction, the reacting molecules come onto the surface of the catalyst. Some of them enter into reaction to give the product, while a few of them get adsorbed on the surface, which is marked by the change in the colour of the catalyst. The fact that the reactant molecules are weakly adsorbed is evident from the catalyst regaining its original colour, when treated with ethanol. The possibility of molecules entering interstices cannot be ruled out. This is observed from the fact that the yields go down after every regeneration, leading to deactivation of the catalyst.

\section{Conclusions}

The work outlined herein reveals the promising use of 12TPA-MCM-41-20 and Al-MCM-41-30 as solid acid 
catalysts in the synthesis of mono esters, the advantages being operational simplicity, where mono esters formed can be simply distilled over, there is no catalyst contamination in products formed, no acid waste generation, the catalysts can be regenerated and reused, and products formed are colourless, a limitation in the conventional process.

\section{Acknowledgement}

The authors are thankful to Sud-Chemie India Pvt. Ltd. for providing instrumentation facilities.

\section{Electronic Supplementary Material}

Supplementary material pertaining to this article is available on the Bulletin of Materials Science website (www.ias.ac.in/matersci).

\section{References}

Aziz A Said A El, Abd El Wahab M M M and Alian A M 2007 J. Chem. Tech. Biotechnol. 82513
Beck J S, Kresge C T, Leonowicz M E, Roth W J and Vartuli J C 1992 Nature 359710

Dong S, Zhu M and Dai B 2012 Green Sust. Chem. 28

Edler K J and White J W 1997 Chem. Mater. 91226

Helen L N, Nicholas A, Zafiropoulos, Thomas F A, Edward T, Samulski D and Wenbin L $2010 \mathrm{~J}$. Am. Oil Chem. Soc. 87 445

Jermy B R and Pandurangan A 2005 Appl. Catal. A: Gen. 295 185

Juan J C, Zhang J and Yarmo M A 2007 J. Mol. Cat. 267265

Kozhevnikov V I, Sinnema A, Jansen R J, Pamin K and Van Bekkum H 1995 Catal. Lett. 30241

Ma Y, Jin T, Wang Z and Li T 2003 Indian J. Chem. 42B 1777

Ma Y, Wang Q L, Jian W and Zuo B 1997 Appl. Catal. A: Gen. 165199

Nascimento L A S, Tito L M Z, Angelina R S, Costa C E F, Zamian J R and Rocha G N 2011 Appl. Catal. B: Environ. 101495

Otera J 2003 Esterification-Methods, reactions and applications (Weinheim, Germany: Wiley-VCH GmbH \& Co. KGaA)

Sharath K R, Vijayshree S and Nagaraju N 2001 Indian J. Chem. Tech. 8362

Srilatha K, Kumar R, Prabhavathi Devi B L A, Prasad R B N, Sai Prasada P S and Lingaiah N 2011 Catal. Sci. Technol. 1662

Xia Q H, Hidajat K and Kawi S 2000 Mater. Lett. 42102

Yadav G and Murkute A D 2003 Int. J. Chem. Reactor Eng. 11

Zhao Z H 2001 J. Mol. Catal. 168147 